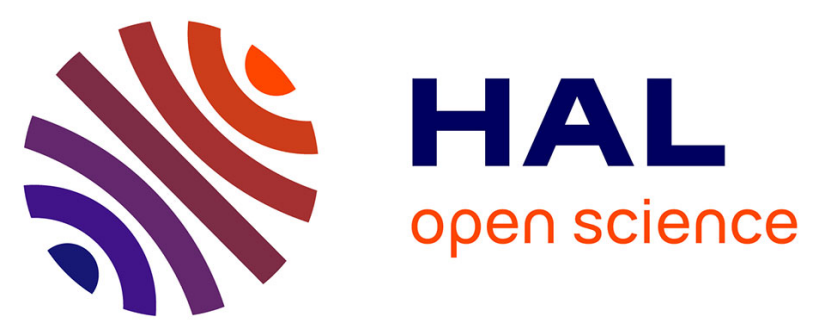

\title{
Étude comparative de la composition et de la contamination des laits des espèces laitières bovines, ovines et caprines. I. ANNEES 1972-1973-1974 - LAITS DE PRODUCTEURS. ISERE: vaches et chèvres. AVEYRON: vaches, chèvres et brebis
}

H. Mahieu, J. C. Le Jaouen, F. M. Luquet, L. Mouillet

\section{To cite this version:}

H. Mahieu, J. C. Le Jaouen, F. M. Luquet, L. Mouillet. Étude comparative de la composition et de la contamination des laits des espèces laitières bovines, ovines et caprines. I. ANNEES 1972-1973-1974 - LAITS DE PRODUCTEURS. ISERE: vaches et chèvres. AVEYRON: vaches, chèvres et brebis. Le Lait, 1977, 57 (565_566), pp.287-300. hal-00928757

\section{HAL Id: hal-00928757 https://hal.science/hal-00928757}

Submitted on 1 Jan 1977

HAL is a multi-disciplinary open access archive for the deposit and dissemination of scientific research documents, whether they are published or not. The documents may come from teaching and research institutions in France or abroad, or from public or private research centers.
L'archive ouverte pluridisciplinaire HAL, est destinée au dépôt et à la diffusion de documents scientifiques de niveau recherche, publiés ou non, émanant des établissements d'enseignement et de recherche français ou étrangers, des laboratoires publics ou privés. 


\title{
Étude comparative de la composition et de la contamination des laits des espèces laitières bovines, ovines et caprines
}

\author{
par \\ H. MAHIEU*, J.C. LE JAOUEN**, F. M. LUQUET*** \\ et L. MOUILLET $* * *$ \\ PREMIÈRE PARTIE \\ ANNEES 1972-1973-1974 - LAITS DE PRODUCTEURS \\ ISÈRE : vaches et chèvres \\ AvEYRON : vaches, chèvres et brebis
}

\section{TABLE DES MATIERES}

INTRODUCTION

I. Modalités DE L'ENQuête

II. ETUdE COMPARATIVE DE LA CONTAMINATION DES LAITS PAR LES RÉSIDUS DE PESTICIDES ORGANOCHLORÉS

H.C.H. $\alpha, \beta$ et $\gamma$

Epoxyde de l'heptachlore et dieldrine

D.D.E. et H.C.B.

III. ETUdE COMPARATIVE DE LA COMPOSITION DES LAITS

Extrait sec

Matières grasses

Matières azotées

Nitrites - Nitrates et urée

\footnotetext{
* I.T.E.B., 149, rue de Bercy - 75579 Paris cedex 12.

** I.T.O.V.I.C., 149, rue de Bercy - 75579 Paris cedex 12.

*** Labcodral, 13, rue de l'Université - 59509 Douai.
} 
IV. ETUdE COMPARATIVE DE LA COMPOSITION MINÉRALE DES LAITS

$\begin{array}{ll}\text { Oligoéléments : } & \text { Cuivre } \\ & \text { Fer } \\ & \text { Manganèse } \\ & \text { Zinc }\end{array}$

Eléments de pollution : Mercure

Plomb

Fluor

Arsenic

Cadmium

CONCLUSION

Chrome

Suite à la série d'observations faites en 1970 et 1971, par la Section lait de l'I.T.E.B., pour la détermination des causes de la présence de résidus de pesticides dans les produits laitiers, il a été demandé que le domaine de ces observations soit élargi et approfondi.

C'est pourquoi il était intéressant de réaliser une étude comparative des laits des espèces bovines, ovines et caprines dans le département de l'Aveyron, où les trois espèces cohabitent ; et de comparer les compositions et les contaminations des laits de vache et de chèvre, dans le département de l'Isère, où des résultats inexplicables avaient été obtenus.

\section{MODALITES DE L'ENQUETE}

Un ingénieur de l'I.T.E.B. a été basé à Lyon pour la durée de cette étude. Il a été chargé d'assurer la mise en place des divers éléments d'enquête et d'assurer le prélèvement et le transport des échantillons jusqu'au laboratoire de Douai.

Une préenquête a été organisée en avril-mai 1972 afin de déterminer les producteurs définitivement retenus.

Toutes les habitudes afférentes aux méthodes culturales et d'élevages du département ont été soigneusement observées. Les problèmes posés par l'utilisation de tout corps chimique sur la ferme (pesticides, engrais, etc.) ont été particulièrement suivis, surtout dans le cas des produits de substitution.

Une visite a été effectuée chez chacun des producteurs retenus. Un questionnaire ayant trait à la marche de l'exploitation et particulièrement à l'utilisation de produits, a été renseigné. L'ingénieur présent sur place, a pu intervenir tout au long de l'enquête pour permettre d'énoncer plus sûrement des interprétations aux phénomènes dépistés. 
Initialement, en 1972, seule l'étude de la contamination était prévue. Il a été demandé qu'une étude relativement poussée de la composition du lait soit effectuée en 1973 et 1974.

L'échantillonnage se présentait comme indiqué dans le tableau 1.

Les races dominantes sont : dans l'Isère, la Montbéliarde et l'Abondance pour les bovins et la Saanen pour les caprins ; dans l'Aveyron, la F.F.P.N. pour les bovins, l'Alpine pour les caprins et la Lacaune pour les ovins.

Pour l'ensemble des espèces, deux prélèvements par an ont été réalisés simultanément :

En 1972 : avril-mai et octobre.

En 1973 : avril et septembre.

En 1974 : avril et octobre.

TABLEAU 1. - Echantillonnage

\begin{tabular}{|c|c|c|c|c|c|c|}
\hline & \multicolumn{3}{|c|}{ Isère } & \multicolumn{3}{|c|}{ Aveyron } \\
\hline & $\begin{array}{l}\text { Non } \\
\text { produc- } \\
\text { teurs }\end{array}$ & $\begin{array}{l}\text { e de } \\
\text { animaux } \\
\text { par } \\
\text { troupeau }\end{array}$ & $\begin{array}{l}\text { Production } \\
\text { annuelle } \\
\text { par animal } \\
\text { (litres) }\end{array}$ & $\begin{array}{l}\text { Nor } \\
\text { produc- } \\
\text { teurs }\end{array}$ & $\begin{array}{l}\text { e de } \\
\text { animaux } \\
\text { par } \\
\text { troupeau }\end{array}$ & $\begin{array}{l}\text { Production } \\
\text { annuelle } \\
\text { par animal } \\
\text { (litres) }\end{array}$ \\
\hline Bovins & 20 & 12 & 3350 & 10 & 32 & 4060 \\
\hline Caprins & 6 & 42 & 625 & 5 & 53 & 475 \\
\hline Ovins & - & - & - & 8 & 217 & - \\
\hline
\end{tabular}

Les échantillons ont été prélevés dans des flacons de $500 \mathrm{~cm}^{3}$ en verre bouché émeri, préalablement bichromatés. La prise d'échantillon, le groupage et le transport jusqu'au laboratoire, ont été assurés par M. Redon, ingénieur à l'I.T.E.B.

Les analyses ont été effectuées au Laboratoire d'Industrie Laitière de l'E.N.S.I.A. à Douai.

Les observations rassemblées sur l'utilisation des produits ont permis de déterminer les causes de la contamination du lait par les 
résidus de pesticides organochlorés ; elles ont fait l'objet d'une publication à part*.

Nous n'abordons dans ce compte rendu que les résultats obtenus sur les laits des élevages suivis durant ces trois années ; ce qui nous permet l'établissement de tableaux de comparaison entre la composition et la contamination des laits des races bovines, ovines et caprines, sur une période suffisamment longue pour éviter des interférences possibles dues à une année particulière.

\section{ETUDE COMPARATIVE DE LA CONTAMINATION DES LAITS PAR LES RESIDUS DE PESTICIDES ORGANOCHLORES}

Sur chaque échantillon, les substances organochlorées suivantes ont été recherchées :

- H.C.H., isomères $\alpha, \beta$ et $\gamma$ (lindane).

- Dieldrine (aldrine).

- Epoxyde de l'heptachlore (heptachlore).

- D.D.E. (D.D.T.-D.D.D.).

- H.C.B.

Les moyennes arithmétiques des résultats sont rassemblées dans le tableau 2 pour le département de l'Isère et dans le tableau 3 pour le département de l'Aveyron.

\section{Résultats et commentaires}

\section{Département de I'Isère}

Les troupeaux bovins sont plus contaminés que les troupeaux caprins ; cela peut venir du fait que les premiers se trouvaient être les plus proches géographiquement des usines chimiques.

La contamination des laits en H.C.H. $(\alpha+\beta)$ est très importante. Pour les bovins elle diminue cependant fortement dans les prélèvements de la sortie de l'hiver ; mais elle reste stable pour les prélèvements effectués au mois d'octobre. Ceci semblerait montrer une plus grande influence de l'environnement que des facteurs propres à l'exploitation (élimination progressive de la contamination par le blanchiment et les traitements sur l'exploitation ; mais des prélèvements de la végétation de surface ont montré une certaine pollution).

* Les sources de contamination du lait par les résidus de pesticides organochlorés, novembre 1973, Institut Technique de l'Elevage Bovin, 149, rue de Bercy, Paris $12^{\mathrm{e}}$. 
TABLEAU 2

Etude comparative de la contamination des laits dans le département de l'Isère (en $\mu \mathrm{g} / \mathrm{kg}$ de matière grasse pure)

\begin{tabular}{|c|c|c|c|c|c|c|c|c|c|c|c|c|}
\hline & \multicolumn{6}{|c|}{ Bovins } & \multicolumn{6}{|c|}{ Caprins } \\
\hline & \multicolumn{2}{|c|}{1972} & \multicolumn{2}{|c|}{1973} & \multicolumn{2}{|c|}{1974} & \multicolumn{2}{|c|}{1972} & \multicolumn{2}{|c|}{1973} & \multicolumn{2}{|c|}{1974} \\
\hline & mai & oct. & avril & sept. & avril & oct. & mai & oct. & avril & sept. & avril & oct. \\
\hline H.C.H. $\alpha$ & 452 & 183 & 286 & 286 & 167 & 275 & 100 & 33 & 72 & 145 & 63 & - \\
\hline H.C.H. $\beta$ & 241 & 161 & 290 & 155 & 70 & 140 & 138 & 63 & 275 & 183 & 47 & - \\
\hline H.C.H. $\alpha+\beta$ & 693 & 344 & 576 & 441 & 237 & 415 & 238 & 96 & 347 & 328 & 110 & - \\
\hline Lindane & 133 & 70 & 126 & 96 & 62 & 102 & 30 & 27 & 72 & 82 & 78 & - \\
\hline H.C.H. total & 826 & 414 & 702 & 537 & 302 & 517 & 268 & 123 & 419 & 410 & 188 & - \\
\hline Epoxyde de l'heptach. & 41 & + & 73 & 40 & 62 & 30 & 30 & 37 & 13 & + & + & - \\
\hline Dieldrine & 108 & 40 & 74 & 75 & 35 & 55 & 33 & 15 & 10 & 23 & + & - \\
\hline D.D.E. & 148 & 259 & 10 & 123 & 21 & 28 & - & + & + & 10 & + & - \\
\hline H.C.B. & 155 & 145 & 160 & 151 & 114 & 175 & 45 & 47 & 125 & 67 & 58 & - \\
\hline
\end{tabular}


TABLEAU 3

Etude comparative de la contamination des laits dans le département de l'Aveyron (en $\mu \mathrm{g} / \mathrm{kg}$ de matière grasse pure)

\begin{tabular}{|c|c|c|c|c|c|c|c|c|c|c|c|c|c|}
\hline & \multicolumn{5}{|c|}{ Bovins } & \multicolumn{5}{|c|}{ Caprins } & \multicolumn{3}{|c|}{ Ovins } \\
\hline & \multicolumn{2}{|c|}{1972} & \multicolumn{2}{|c|}{1973} & \multirow{2}{*}{$\begin{array}{r}1974 \\
\text { avril }\end{array}$} & \multicolumn{2}{|c|}{1972} & \multicolumn{2}{|c|}{1973} & \multirow{2}{*}{$\begin{array}{c}1974 \\
\text { avril }\end{array}$} & \multirow{2}{*}{$\begin{array}{l}1972 \\
\text { mai }\end{array}$} & \multirow{2}{*}{$\begin{array}{c}1973 \\
\text { avril }\end{array}$} & \multirow{2}{*}{$\begin{array}{l}1974 \\
\text { avri }\end{array}$} \\
\hline & mai & oct. & avril & sept. & & mai & oct. & avril & sept. & & & & \\
\hline H.C.H. $\alpha$ & 63 & 84 & 43 & 79 & 55 & 28 & 35 & 61 & 74 & 46 & 32 & 54 & 72 \\
\hline H.C.Н. $\beta$ & 53 & 34 & 71 & 31 & 40 & 41 & 115 & 63 & 84 & 46 & 120 & 44 & 40 \\
\hline H.C.H. $\alpha+\beta$ & 116 & 118 & 114 & 110 & 95 & 69 & 150 & 124 & 158 & 92 & 152 & 98 & 112 \\
\hline Lindane & 23 & 78 & 65 & 67 & 65 & 23 & 20 & 60 & 328 & 159 & 40 & 38 & 100 \\
\hline H.C.H. total & 139 & 196 & 179 & 177 & 160 & 92 & 170 & 184 & 486 & 251 & 192 & 136 & 212 \\
\hline Epoxyde de l'heptach. & 23 & + & + & 31 & 68 & 15 & + & ++ & 50 & ++ & 26 & + & 35 \\
\hline Dieldrine & 34 & ++ & 63 & 24 & ++ & 18 & + & ++ & 18 & ++ & 10 & + & + \\
\hline D.D.E. & 51 & + & ++ & + & ++ & 50 & + & ++ & 14 & 127 & 100 & + & + \\
\hline H.C.B. & 22 & 70 & 29 & 28 & 53 & 27 & 20 & 62 & 64 & 74 & 36 & 58 & 55 \\
\hline
\end{tabular}


Ceci est d'ailleurs confirmé par la surprenante stabilité de la contamination par le H.C.B. qui n'était pas utilisé dans les exploitations enquêtées.

Pour les caprins, on observe une importante contamination des laits en 1973 ; la teneur des laits en résidus de H.C.H. $\alpha+\beta$ revient à une valeur acceptable en avril 1974, bien qu'encore légèrement supérieure aux recommandations les plus sévères $(100 \mu \mathrm{g} / \mathrm{kg})$.

Pour les autres résidus de pesticides organochlorés, après une présence certaine en 1972 et en 1973, il n'en a été décelé que des traces relativement faibles en 1974.

Les objets de préoccupation pour ce département restent donc la présence dans le lait de résidus de H.C.H. $(\alpha+\beta)$ et dans une moindre mesure de H.C.B.

\section{Département de l'Aveyron}

La contamination des laits des trois espèces par des résidus de H.C.B. est faible, mais relativement constante et de niveau comparable.

Celle par l'époxyde de l'heptachlore, la dieldrine et le D.D.E. est négligeable et sporadique. Il faut cependant observer les $127 \mu \mathrm{g} / \mathrm{kg}$ de matière grasse pure de D.D.E. chez les caprins en avril 1974.

Par contre la présence des résidus de H.C.H. dans les laits des trois espèces, bien que faible reste toutefois proche des recommandations du marché extérieur $(100 \mu \mathrm{g} / \mathrm{kg}$ de matière grasse pure pour le H.C.H. $\alpha+\beta$ et $200 \mu \mathrm{g} / \mathrm{kg}$ de matière grasse pure pour le lindane).

- Bovins : pour les cinq prélèvements, la contamination est d'une grande régularité et présente une légère et continuelle décroissance. Ceci semble montrer que l'on est arrivé au stade de la décroissance résiduelle.

- Caprins : la contamination par les résidus de H.C.H. $\alpha+\beta$, importante en 1972-1973, est en forte diminution en 1974 ; par contre la présence de résidus de lindane est préoccupante en septembre 1973 et à un moindre degré en avril 1974.

- Ovins : la contamination par les résidus de H.C.H. $\alpha+\beta$ est importante en 1972 et s'est stabilisée à des doses admissibles en 1973 et 1974 ; par contre la présence de résidus de lindane a doublé en 1974 par rapport aux deux années précédentes.

Dans ce département, malgré quelques contaminations sporadiques, le problème posé par la présence de résidus de pesticides organochlorés dans le lait semble résolu. Cependant, il faut dans J'avenir rester vigilant afin que des incidents comme celui arrivé chez les chèvres en septembre 1973 (H.C.H.) ne se reproduisent pas. 


\section{ETUDE COMPARATIVE DE LA COMPOSITION DES LAITS}

Trois séries de prélèvements ont généralement été effectuées ; exception faite pour les bovins de l'Isère où un échantillonnage supplémentaire est intervenu en octobre 1974.

Sur chaque échantillon les déterminations suivantes ont été effectuées :

Extrait sec total.

Matières grasses.

Matières azotées totales : — Kjeldahl.

- Noir amido.

Caséines.

Protéines du sérum.

Protéines.

Azote non-protéine (N.P.N.).

Urée.

Nitrates - Nitrites.

Les matières azotées du lait ont été différenciées en utilisant la méthode de Rowland: les analyses des azotes non-caséines et des azotes non-protéines ont été effectuées à l'aide de l'appareil Technicon. Le coefficient appliqué a été celui de 6,39; et les teneurs en caséines, protéines du sérum et protéines ont été calculées par différence en utilisant les résultats donnés par les analyses directes du N.P.N. et de la matière azotée totale.

Les moyennes arithmétiques des résultats sont rassemblées dans le tableau 4 pour le département de l'Isère et dans le tableau 5 pour le département de l'Aveyron.

\section{Composition des laits dans le département de l'Isère}

Le lait de chèvre est moins riche en extrait sec que le lait de vache. Chaque élément semble participer de façon proportionnelle : environ trois points pour la matière grasse et pour la matière azotée ; pour cette dernière la différence se joue surtout au niveau des caséines. Par contre, le lait de chèvre est proportionnellement deux fois plus riche en N.P.N. que le lait de vache.

Pour les deux espèces les laits de septembre 1973 sont plus riches en matières grasses et en matières azotées que les laits d'avril 1974 (environ deux points) ; pour ces dernières la différence est surtout sensible au niveau des caséines.

Il semble que le lait de chèvre soit plus riche en urée que le lait de vache. Il n'y a pas de nitrites dans les laits des deux espèces. Les nitrates sont absents du lait, au sortir de la mamelle, leur pré- 


\section{TABLEAU 4}

Composition des laits dans le département de l'Isère (unité : g/l)

\begin{tabular}{|c|c|c|c|c|c|c|c|}
\hline & \multicolumn{4}{|c|}{ Bovins } & \multicolumn{3}{|c|}{ Caprins } \\
\hline & & & 19 & & 19 & & 1974 \\
\hline & avril & sept. & avril & oct. & avril & sept. & avril \\
\hline Extrait sec & 127,6 & 127,7 & 123,2 & 134,2 & 115,4 & 115,4 & 117,4 \\
\hline Matières grasses & 35,9 & 36,6 & 33,4 & 39,2 & 32,2 & 32,1 & 30,9 \\
\hline Extrait sec dégraissé & 91,7 & 91,1 & 89,8 & 95,0 & 83,2 & 83,3 & 86,5 \\
\hline $\begin{array}{l}\text { Matières azotées tot. : } \\
\text { Kjeldahl } \\
\text { Noir amido }\end{array}$ & $\overline{-}$ & $\overline{33,9}$ & $\begin{array}{l}31,8 \\
32,2\end{array}$ & $\begin{array}{l}35,2 \\
35,3\end{array}$ & $\overline{28,1}$ & $\overline{30,8}$ & $\begin{array}{l}28,2 \\
28,0\end{array}$ \\
\hline Caséines & - & 28,3 & 24,6 & 26,8 & 18,9 & 23,4 & 20,7 \\
\hline Protéines du sérum & - & 4,3 & 5,4 & 6,0 & 5,2 & 5,1 & 4,1 \\
\hline Protéines & - & 32,6 & 30,0 & 32,8 & 24,1 & 28,5 & 24,8 \\
\hline N.P.N. & - & 1,44 & 1,77 & 1,88 & 3,00 & 2,29 & 3,13 \\
\hline
\end{tabular}

(unité : mg/l)

\begin{tabular}{l|c|c|c|c|c|c|c}
\hline Urée & 183 & 88 & 244 & 241 & 296 & 178 & 420 \\
Nitrites & 0 & 0 & 0 & 0 & 0 & 0 & 0 \\
Nitrates & - & 0,18 & 0,21 & - & 0,36 & 0,35 & 0,30 \\
& & & & & & & \\
\hline
\end{tabular}

sence ne traduit donc qu'une contamination plus ou moins importante après la traite.

\section{Composition des laits dans le département de l'Aveyron}

Le lait de brebis est de loin le plus riche en extrait sec ; et celui de chèvre est le moins riche. Contrairement à ce que l'on observe 
dans le département de l'Isère les laits de chèvre et de vache sont plus riches en matières grasses et moins riches en matières azotées en septembre 1973 qu'en avril 1974 ; la différence dans ce dernier cas étant due exclusivement au N.P.N.

Il apparaît dans le tableau 5 que le lait de brebis est le plus riche et celui de vache le moins riche en urée ; le lait de chèvre se situant à une valeur médiane. La contamination du lait par les nitra-

TABLEAU 5

Composition des laits dans le département de l'Aveyron (unité : $\mathrm{g} / \mathrm{l}$ )

\begin{tabular}{|c|c|c|c|c|c|c|c|}
\hline & \multicolumn{3}{|c|}{ Bovins } & \multicolumn{3}{|c|}{ Caprins } & \multirow{3}{*}{$\begin{array}{c}\text { Ovins } \\
1974 \\
\text { avril }\end{array}$} \\
\hline & \multicolumn{2}{|c|}{1973} & 1974 & \multicolumn{2}{|c|}{1973} & 1974 & \\
\hline & avril & sept. & avril & avril & sept. & avril & \\
\hline Extrait sec & 132,1 & 124,7 & 127,9 & 114,2 & 116,2 & 116,5 & 180,9 \\
\hline Matières grasses & 41,1 & 37,1 & 36,0 & 36,8 & 33,1 & 31,3 & 71,7 \\
\hline Extrait sec dégraissé & 91,0 & 87,6 & 91,9 & 77,4 & 83,1 & 85,2 & 109,2 \\
\hline $\begin{array}{l}\text { Matières azotées tot. : } \\
\text { Kjeldahl } \\
\text { Noir amido }\end{array}$ & $\overline{32,2}$ & $\overline{32,1}$ & $\begin{array}{l}32,8 \\
33,0\end{array}$ & $\overline{27,6}$ & $\overline{27}_{27}$ & $\begin{array}{l}29,1 \\
28,4\end{array}$ & $\begin{array}{l}53,8 \\
52,4\end{array}$ \\
\hline Caséines & 25,2 & 24,3 & 25,0 & 18,7 & 20,3 & 20,0 & 40,2 \\
\hline Protéines du sérum & 5,9 & 5,4 & 5,6 & 5,6 & 4,6 & 4,5 & 9,0 \\
\hline Protéines & 31,1 & 29,7 & 30,6 & 24,3 & 24,9 & 24,5 & 49,2 \\
\hline N.P.N. & 1,66 & 1,67 & 1,75 & 3,32 & 2,25 & 3,87 & 3,17 \\
\hline
\end{tabular}

(unité : mg/l)

\begin{tabular}{l|r|r|r|r|r|r|r}
\hline Urée & 190 & 212 & 256 & 309 & 90 & 402 & 530 \\
Nitrites & 0 & 0 & 0 & 0 & 0 & 0 & 0 \\
Nitrates & - & 0,37 & 0,15 & - & 0,31 & 0,35 & - \\
& & & & & & & \\
\hline
\end{tabular}


tes après la traite, semble être identique chez les ovins et les caprins dans ce département.

\section{ETUDE COMPARATIVE DE LA COMPOSITION MINERALE DES LAITS}

Cette étude n'a pu être réalisée totalement car seuls les laits de vache dans l'Isère ont été analysés en septembre 1973 ; et les laits de chèvre et de brebis dans l'Aveyron en avril 1973.

Les résultats enregistrés sont répartis dans le tableau 6.

TABLEAU 6. - Analyse minérale des laits (unité : $\mu \mathrm{g} / \mathrm{l}$ )

\begin{tabular}{l|c|c|c}
\hline & Isère (sept. 73) & \multicolumn{2}{|c}{ Aveyron (avril 73) } \\
\cline { 2 - 3 } & Bovins & Caprins & Ovins \\
\cline { 2 - 3 } Fer & 706 & 750 & 598 \\
Cuivre & 76 & 50 & 52 \\
Manganèse & 31 & 20 & 68 \\
Zinc & 1491 & 600 & 1440 \\
Mercure & - & - & - \\
Plomb & - & - & 25 \\
Fluor & 23 & 19 & 10 \\
Arsenic & 50 & - & 31 \\
Cadmium & 28 & ++ & ++ \\
Chrome & - & & 22 \\
& & & \\
\hline
\end{tabular}

Une étude sur la teneur des laits individuels a été effectuée sur le cheptel laitier bovin en septembre 1973 (cf. publication I.T.E.B. Labcodral, septembre 1976 : à propos de la teneur des laits individuels et de mélange en matières minérales et urée). 


\section{TABLEAU 7}

Récapitulatif des résultats observés sur les minéraux en septembre 1973 (lait de vache $-\mu \mathrm{g} / \mathrm{l}$ )

\begin{tabular}{l|rr|c} 
& $\begin{array}{c}\text { Valeurs données } \\
\text { par la littérature }\end{array}$ & $\begin{array}{c}\text { Moyennes arithmétiques } \\
(1973)\end{array}$ \\
\cline { 2 - 3 } Fer & 100 à & 250 & 1019 \\
Cuivre & 20 à̀ & 40 & 122 \\
Manganèse & 20 à & 50 & 45 \\
Zinc & 2000 à 5000 & 2680 \\
Mercure & 6 à & 10 & - \\
Plomb & 40 à & 100 & - \\
Fluor & 5 à̀ & 25 & 14 \\
Arsenic & 30 à̀ & 50 & 59 \\
Cadmium & 1 à̀ & 10 & 34 \\
& & & \\
\hline
\end{tabular}

Pour les brebis on peut trouver dans la littérature quelques données :

$\begin{array}{lrr}\text { Fer } & 500-700 \mu \mathrm{g} / 1 . \\ \text { Cuivre } & 100-200 \mu \mathrm{g} / \mathrm{l} . \\ \text { Zinc } & 3000-6000 \mu \mathrm{g} / 1 .\end{array}$

En comparant les tableaux précédents l'on peut dire que :

- Les teneurs des laits des différentes espèces en fer et en cuivre traduisent plus une contamination des laits après la traite que la teneur réelle au sortir de la mamelle.

- La teneur des laits en manganèse est plus faible chez les caprins, que chez les ovins ; chez ces derniers la teneur semble plus élevée que celle indiquée dans la littérature, pour les vaches.

- La teneur des laits en zinc est particulièrement faible dans les deux départements concernés ; le lait de chèvre semblant particulièrement pauvre.

- Aucune contamination du lait par le mercure ou le plomb n'a pu être constatée. 
- Les contaminations par le fluor et l'arsenic sont conformes aux données trouvées dans la littérature; elles sont semblables pour le fluor ; la présence d'arsenic semble élevée dans l'Isère mais elle est inférieure à la moyenne nationale des résultats obtenus en septembre 1973.

- La contamination par le cadmium semble élevée au regard de la norme allemande récemment publiée $(10 \mu \mathrm{g} / 1)$; mais il n'y a pas lieu de s'alarmer encore actuellement.

\section{象}

La recherche des cellules dans les laits des différentes espèces, a été faite systématiquement par comptage au Coulter Counter (tab. 8).

TABLEAU 8

Nombre de cellules dans le lait - Résultats moyens

\begin{tabular}{l|c|c}
\hline & Aveyron & Isère \\
\cline { 2 - 3 } Bovins & 568000 & \\
Caprins & 990000 & 232700 \\
Ovins & 400500 & 672500 \\
& & - \\
\hline
\end{tabular}

Si la présence des cellules est relativement faible dans l'Isère, elle serait plus inquiétante dans l'Aveyron où la moyenne des troupeaux indiquerait un plus grand niveau de perte dû aux mammites. Une étude de Roguinsky a en effet montré que les normes applicables aux bovins (et aux ovins) ne le sont pas aux caprins, et que, au seuil de 500000 cellules chez les bovins, correspondrait celui de 1000000 de cellules chez les caprins.

\section{CONCLUSION}

Bien que limitée à deux départements (Isère et Aveyron) cette enquête sur des laits de producteurs a permis d'aborder un problème difficile à résoudre sur le terrain : l'étude approfondie de la composition des laits. 
Elle a permis, en outre, de préciser les méthodes de prélèvement, de transport d'échantillons, d'analyse pour chacun des éléments étudiés.

Cette étude comparative a été prolongée en 1974 sur le lait de grand mélange dans six laiteries effectuant simultanément le double ramassage.

\section{Remerciements}

Nous tenons à remercier :

- Le laboratoire qui a mis au point les méthodes d'extraction appropriées et effectué les analyses (Labcodral, Douai).

- Les laiteries et syndicats qui ont participé à cette enquête :

- pour l'Isère:

ORLAC, usine de Sassenage,

Syndicat Caprin de l'Isère,

- pour l'Aveyron:

Fromageries des Gorges du Tarn,

Coopérative Agricole Laitière Aveyronnaise,

Coopérative des Gorges du Lot - Entraygues (fromage de chèvre),

Confédération Générale de Roquefort.

- Les producteurs qui ont accepté de répondre aux questionnaires et ont fourni le lait des prélèvements.

Reçu pour publication en mars 1977. 